\title{
MAGNETIC FIELDS IN THE SPIRAL GALAXY NGC 6946
}

\author{
MATTHIAS EHLE, RAINER BECK \\ Max-Planck-Institut für Radioastronomie, Auf dem Hügel 69, W-5300 Bonn 1, FRG
}

December 2, 1992

\begin{abstract}
High frequency polarization observations reveal the existence of a large-scale ordered magnetic field in the disk of the spiral galaxy NGC 6946. At lower frequencies the disk is no longer transparent to polarized radio waves due to Faraday depolarization. The spiral pattern of the uniform magnetic field and the distribution of polarized intensities are fairly well simulated by a dynamo model. The model parameters indicate that the dynamo does not only operate in the disk, but also in the halo.
\end{abstract}

\section{Observations and Results}

VLA observations at $\lambda 18.0 \mathrm{~cm}$ and $\lambda 20.5 \mathrm{~cm}$ (Beck, 1991) show strong Faraday depolarization with only small Faraday depths. Hence only uniform magnetic fields in the upper disk or halo are observable. On the contrary, Effelsberg observations at $\lambda 2.8 \mathrm{~cm}$ (Ehle, 1991) allow to look through the whole plane; the magnetic field seems to closely follow the optical spiral arms even in regions with largely varying pitch angles (Fig. 1). We calculated the azimuthal variation of rotation measures (RM) in the plane of the galaxy (Fig. 2). We estimated a constant offset, due to foreground Faraday rotation, of $36 \mathrm{rad} \mathrm{m}^{-2}$. Internal Faraday rotation between $\lambda 18.0 \mathrm{~cm}$ and $\lambda 20.5 \mathrm{~cm}$ is generally low due to depolarization (Fig. 2, bottom). A few high RM values indicate vertical field lines.
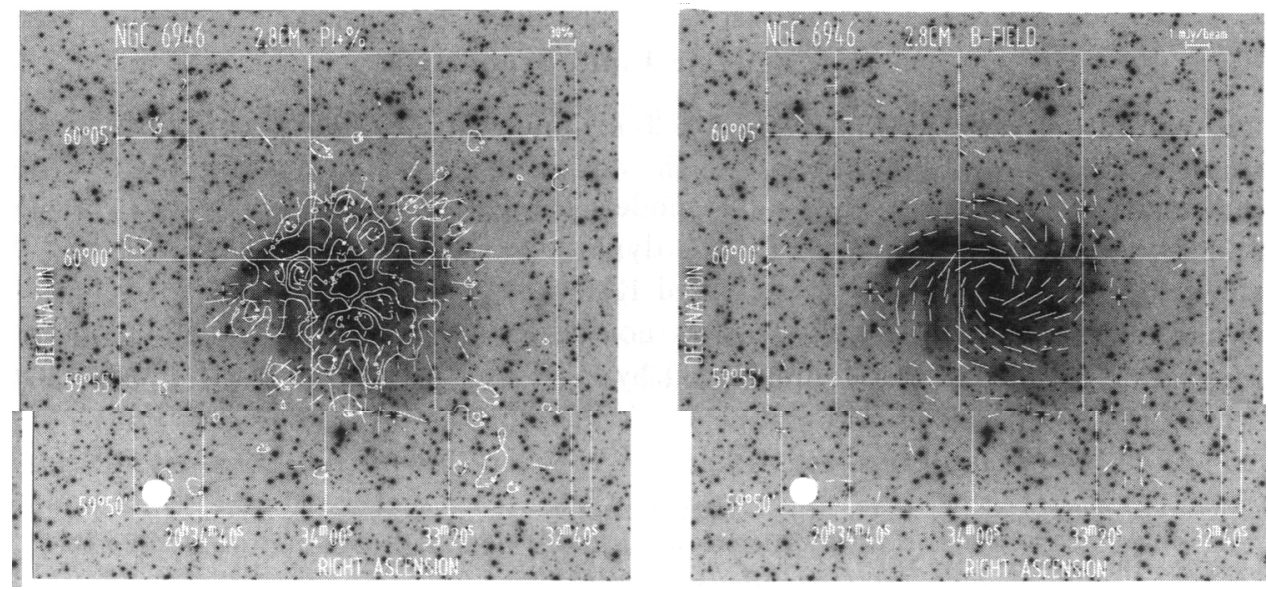

Fig. 1. The polarized intensity map (left) and intrinsic magnetic field orientation (right) of NGC 6946 at $\lambda 2.8 \mathrm{~cm}$ from Ehle and Beck (1993) 


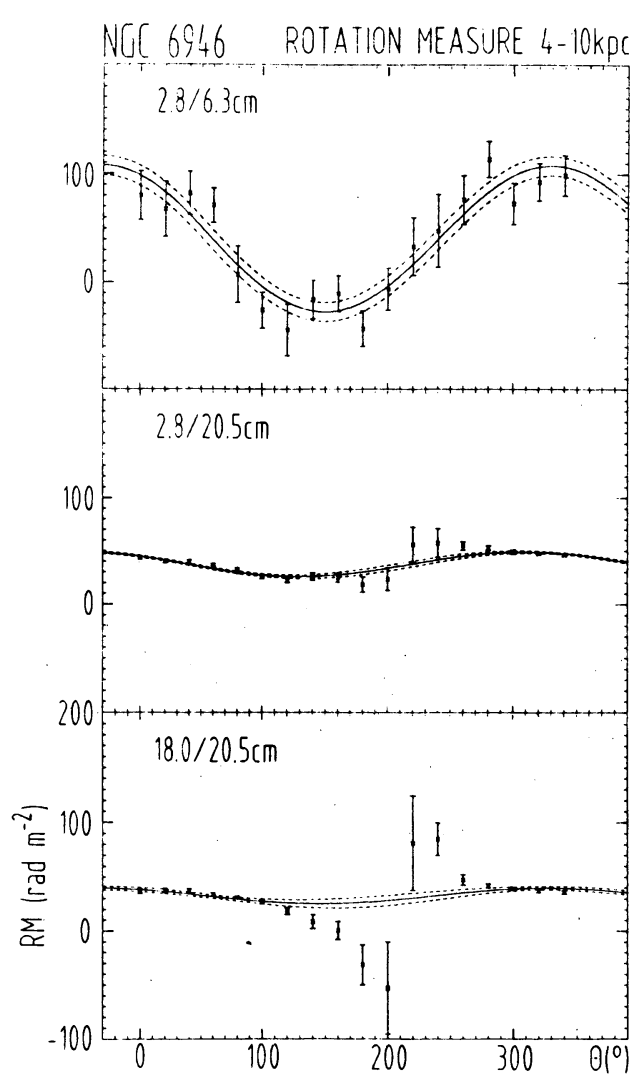

Fig. 2.
Between $\lambda 2.8 \mathrm{~cm}$ and $\lambda 6.3 \mathrm{~cm}$ the galaxy is transparent for polarized radio emission, and high rotation measures with $77 \pm 6 \mathrm{rad} \mathrm{m}^{-2}$ amplitude are observed (Fig. 2, top). This amplitude requires an average electron density of $\simeq 0.27 \mathrm{~cm}^{-3}$ in the plane of the galaxy $(100 \mathrm{pc}$ full thickness) and an intrinsic strength of the uniform field of $\simeq 12 \mu \mathrm{G}$; both numbers are significantly higher than in our Galaxy. At longer wavelengths strong Faraday depolarization in a turbulent medium makes the galaxy "optically thick" for polarized radio emission. Our data give evidence for the existence of small clumps of ionized gas ( $\simeq 1 \mathrm{pc}$ size and $\simeq 5 \mathrm{~cm}^{-3}$ electron density). We propose that the ionized gas has three components with different vertical extensions and with different filling factors: a) The diffuse ionized gas with low electron density, high filling factor and large vertical extent, b) the classical and giant HII regions with high density, low filling factor and small vertical extent, c) the small low-density HII regions with intermediate filling factor. In NGC 6946 component c) seems to dominate.

\section{Magnetic Field Structure}

The azimuthal variations of $\operatorname{RM}(2.8 / 6.3 \mathrm{~cm})$ and $\mathrm{RM}(2.8 / 20.5 \mathrm{~cm})$ (Fig. 2a,b) are singly-periodical and give support to the action of a galactic dynamo. An axisymmetric S0 field (Elstner et al., 1992) models the distribution of polarized intensity and field orientations fairly well if the dynamo operates not only in the disk, but also in the halo. However, the observed RM variation is inconsistent with a basic dynamo mode because its phase does not agree with the field pitch angle. The magnetic field appears to be influenced by local effects near the plane (flow of gas out of the plane or a radial component of streaming motions) (Ehle, 1991).

\section{References}

Beck, R.: 1991, $A \mathcal{E} A$ 251, 15

Ehle, M.: 1991, Diploma Thesis, Univ. of Bonn

Ehle, M. and Beck, R.: 1993. $A \mathcal{E} A$ (in press)

Elstner, D., Meinel, R., Beck, R.: 1992, $A \& A S$ 94, 587 